\title{
The Evaluation of Environmental Safety and the Cleanup of Pollutants Using Biological Functions
}

\section{Tamami KAWASAKI}

Assistant Senior Researcher,

\section{Toshio HAYAKAWA}

\section{Minoru SHIMURA}

Kazuhide KIMBARA

Senior Researcher, Assistant Senior Researcher, Senior Researcher, Laboratory Head, Biotechnology G., Environmental Engineering Div.

\begin{abstract}
Evaluation of environmental safety is very important for human health. It is known that railways provide a clean transportation system with low $\mathrm{CO}_{2}$ emission, but there is little information about the evaluation of ecotoxicological safety in the railway transportation system. In this paper, we report about ecotoxicological safety tests and the bioremediation research for PCB contaminated ballast stones. The former is an introduction of biological methods used in our laboratory to evaluate the environmental safety that can be applied to railway environments. The latter is an example of bioremediation research using a PCB degrading microorganism. Since there are no good physico-chemical methods to remove PCB contamination from ballast stones, bioremediation might lead us to a solution for environmental safety.
\end{abstract}

Keywords : environment, safety evaluation, biological function, biodegradation, microorganism, PCB, ballast

\section{Introduction}

Recently, people are becoming more and more concerned about global environmental problems. It is known that railways provide a clean transportation system with low $\mathrm{CO}_{2}$ emission. The Ministry of Land, Infrastructure and Transport of Japan suggests that the volume of railway traffic is likely to increase in the near future, as expressed by the term "modal shift". UIC (International Union of Railways) has just begun to address the environmental loads on railway systems and held a UIC conference on environmental indicators, in 2001 May, to discuss the railway environment ${ }^{1}$. It is necessary to rerecognize the co-existence between nature and railway infrastructures. This paper reports the co-existence of nature and railway environment and the evaluation of environmental loads by using biological systems.

Figure 1 shows a scheme to evaluate the environmental safety using biological systems. First, the toxicity in the environment was evaluated by biological sensitivities. Second, the pollution was cleaned up by using biological degradation ability. Physico-chemical methods are used to analyze and identify pollutants and biological methods are used to evaluate the toxicity. We can evaluate the environmental safety using these two methods totally.

We will also report a bioremediation research for PCB contaminated ballast stones using a PCB degrading microorganism.

\section{Biological ecotoxicological safety test}

There are several types of biological ecotoxicological safety tests to evaluate the effect of environmental pollutants on creatures. These tests evaluate the toxic effect on individual DNAs, cells and organisms.

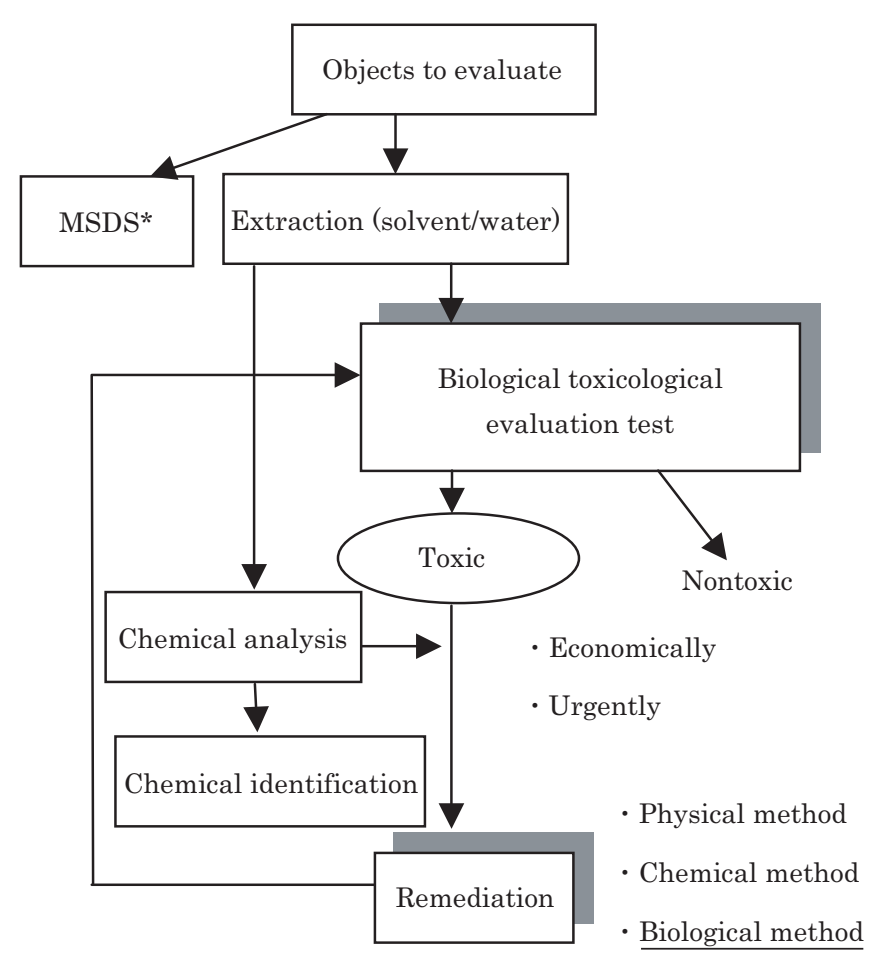

*Material Safety Data Sheet Fig. 1 The flow of environmental safety test with biological
systems

\subsection{Ames test}

The Ames test is a conventional screening method that is widely used to detect the possibility of mutagenecity of chemicals on DNAs. In this test, we can quantify the ability to cause mutation on DNA by targeted chemicals. In our laboratory, this method was used to confirm whether 
magnetic fields have any effect on bioorganisms ${ }^{2) 3)}$. This method was also used to evaluate the mutagenicity of the effluent from the biodegradation system of polychlorinated byphenyl (PCB) that was developed in our laboratory and it is confirmed that the effluent has no mutagenecity.

\subsection{Flow cytometry (FCM) analysis}

FCM is a tool with great potential to perform both qualitative and quantitative analyses, based on the simultaneous measurements of structural and functional parameters of individual cells. These measurements are based on light scatter, which reflects the cell size and structure, and fluorescence which would reflect DNA contents, enzyme activity, respiration, membrane potential or membrane integrity, depending on the fluorescent dye being used. In combination with DNA fluorescent dyes, FCM has been widely used to measure DNA contents and analyze the cell cycle of eukaryotes, prokaryotes and archaea.

Figure 2 shows that the number of microorganisms that grew normally decreased under the presence of PCB in the culture medium ${ }^{4}$. This is the first report on the toxic effect of PCB on the growth of microorganisms. Therefore, it seems that the FCM can be used for environmental monitoring to detect the toxicity of chemicals on the growth of microorganisms.

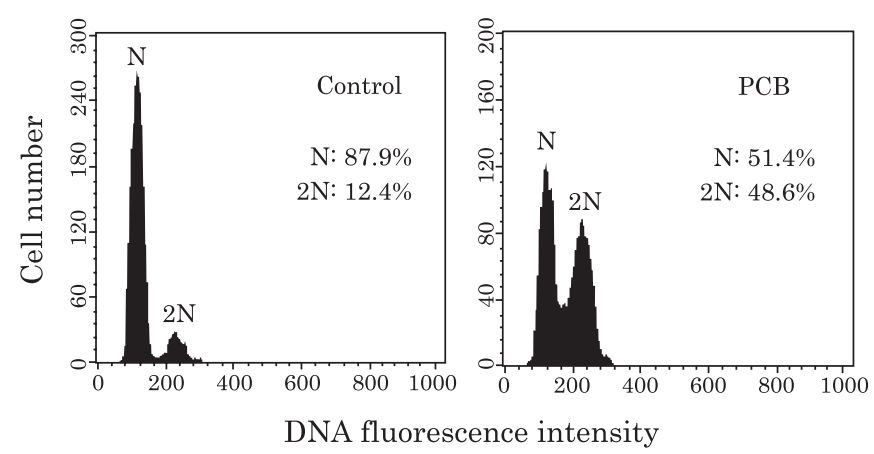

Fig. 2 Detection of PCB effect on microorganisms cell cycle by analyzing with flow cytometry

\subsection{Daphnia toxicological test}

The Daphnia toxicological test is one of the methods that are authorized by OECD and ISO. This method is also recognized internationally as a method to evaluate toxic chemical effects in environments. In this method, the arrest of the movement of Daphnia was measured after chemicals were introduced into the breeding system and the toxicity was evaluated. This method is standardized by Japanese Industrial Standards (JIS). It is known that Daphnia is sensitive to chemicals and easy to keep in the laboratory at low cost. That is the reason why Daphnia is generally used to evaluate toxicity. There are several reports on the evaluation of pollution by adding extracts from contaminated soil to the Daphnia-keeping system $^{5)}$. We are trying to make a model system by using this method to evaluate the toxicity after cleanup of polluted sites.

\subsection{Mutation assay in Tradescantia}

Tradescantia is used for a conventional mutation assay to detect environmental mutagen, because it is known that the violet color of Tradescantias stamen hair changes to pink, which is called pink mutation, after it is exposed to some mutagenic resources. This test system was established in our laboratory and the mutation effect of heavy metals was confirmed by this system. Table 1 shows the increase in the pink mutation frequency caused by $\mathrm{Cu}$, which was added to the liquid medium of Tradescantia as $100 \mathrm{mg} / \mathrm{l}$ copper sulfate. The increase in pink mutation frequency after $\mathrm{Cu}$ treatment was confirmed statistically.

We have a plan to use this detecting system to monitor and evaluate the railway environment in the future.

\section{Table 1 Detection of mutagenecity on Tradascantia pink mutation caused by copper (Cu) Pink mutation Number of stamen cells

\begin{tabular}{ccc} 
Control & 44 & 172,234 \\
Cu treatment & 102 & 257,194 \\
\hline & & ${ }^{*}$ Cu concentration $100 \mathrm{mg} / \mathrm{l}$
\end{tabular}

\section{Trial research for bioremediation for ballast stones}

The soil and ballast along railway lines, which were contaminated with PCB as a result of railway accidents in the past, are still kept untreated and stored as hazardous materials.

It may be difficult to clean PCB contaminated soil and ballast by the existing PCB treatment methods such as chemical decomposition and supercritical water oxidation. In our laboratory, research concerning PCB oil degradation by microorganisms was performed. Our PCB treatment system by biodegradation succeeded in degrading PCB efficiently and safely. Therefore, we applied our PCB biodegradation method to the treatment for PCB contaminated soil and ballast.

\subsection{Washing with solvent}

\subsubsection{Preparing for PCB treated test ballast}

PCB treated ballast stones were prepared as follows. One hundred microliters of ethanol containing $1 \mathrm{mg}$ of Kaneclor 300 (KC 300, mainly consisting of tri-chlorinated PCB congeners) were applied to each ballast stone. These ballast stones were settled in a draft chamber for a week to age the samples and used for further experiments.

\subsubsection{Ultrasonic washing}

The PCB treated ballast samples were washed with hexane in an ultrasonic cleaning bath (Yamato Co., BRANSON 2200). As a result, only about $20 \%$ of PCB were extracted after ultrasonic washing. It seemed that about $80 \%$ of PCB remained on the surface or inside ballast stones. The remaining PCB on ballast stones should be degraded by other methods. 


\subsection{Biodegradation using a PCB degrading microorganism}

Ultrasonic washing with hexane does not seem to completely remove PCB from the ballast stones. In addition, there are no reports that show how to remove PCB chemically from PCB contaminated ballast stones. This is the reason why we used the biodegrading ability of microorganisms to degrade PCB on the surface of or inside ballast stones.

\subsubsection{Characteristic of a PCB degrading microorganism}

A PCB degrading bacterium, Comamonas testosteroni TK102, was used for this experiment. This TK102 strain has an ability to utilize biphenyl as carbon and energy sources and to degrade a portion of 4-chlorinated PCB congeners.

\subsubsection{Microorganism biofilm}

A biofilm is generated by microorganisms on the surface of a material, such as of a ballast stone, when it grows. TK102 is classified as a group of biofilm-making microorganisms. If TK102 can make biofilms on the surface of ballast stones, biodegradation of PCB will be expected thereon.

\subsubsection{Culture conditions}

TK102 was grown on W medium with biphenyl as a carbon source at $30^{\circ} \mathrm{C}$. Cells were grown in $100 \mathrm{ml}$ of the medium containing $500 \mathrm{mg} / \mathrm{l}$ of biphenyl at $30^{\circ} \mathrm{C}$ for 48 hours on a reciprocal shaker $(100 \mathrm{rpm})$ and were used as inoculum $^{6}$. Fifty milliliters of the pre-culture was inoculated into $5 \mathrm{~L}$ of $\mathrm{W}$ medium and incubated at $30^{\circ} \mathrm{C}(300$ rpm). A 10-liter fermentor NTBR type F-10, (Sakura Seiki Co., Japan) was used for this $5 \mathrm{~L}$ culture. $2.4 \mathrm{~L}$ of $5 \mathrm{~L}$ TK102 culture medium was centrifuged $(5,000 \mathrm{rpm})$ for 20 minutes and the cell pellets were collected. The collected cells were resuspended into $400 \mathrm{ml}$ of $\mathrm{W}$ medium, and this medium was added to the treatment apparatus. This cultivation and addition of TK102 was performed once a week.

\subsubsection{Biodegradation of ballast stones in a bioreactor}

A bioreactor was used for the biodegradation of PCB attached ballast stones.

This bioreactor was composed of a plastic cylinder $(\phi$ $20 \mathrm{~cm}$, height $65.5 \mathrm{~cm}$ ) and a suction pump to circulate the culture medium (Figure 3). Forty ballast stones, which were contaminated with PCB, were set into the cylinder and the medium solution containing TK102 was made to constantly circulate within the cylinder by the suction power of the pump. The circulated medium was continually sprinkled over the ballast stones from a nozzle that was set on the top of the cylinder.

\subsubsection{PCB analysis}

Three ballast stones were collected from the bioreactor to analyze the amount of remaining PCB on the surface of the ballast stones. These samples were collected 0,30 , 66, 91 and 132 days after starting the biodegradation.

The remaining $\mathrm{PCB}$ of the ballast stones was extracted with $300 \mathrm{ml}$ of hexane by using an ultrasonic bath for $30 \mathrm{~min}$. After the ultrasonic treatment, solutions were analyzed by gas chromatography (Shimadzu Co., GC-7A).

\subsection{Results}

\subsubsection{Decrease of PCB on the ballast stones}

Figure 4 shows the reduction of PCB on the ballast stones after the treatment using the PCB-degrading microorganism, TK102. According to Figure 4, PCB decreased 54, 73 and $70 \%$ after 30,66 and 132 days, respectively, from the amount on Day 0 . The data also suggest that there was a limit in the efficient degradation of PCB after treatment for 66 days or longer.

\subsubsection{PCB in culture medium}

The PCB in the circulated TK102 medium was also analyzed on the same days of the sampling of the ballast stones. As a result, PCB was not detected in any liquid sample. Since PCB did not exist in the TK102 medium that was circulated in the apparatus, PCB seemed to have been degraded by TK102 that formed biofilms on the surface of ballast stones.
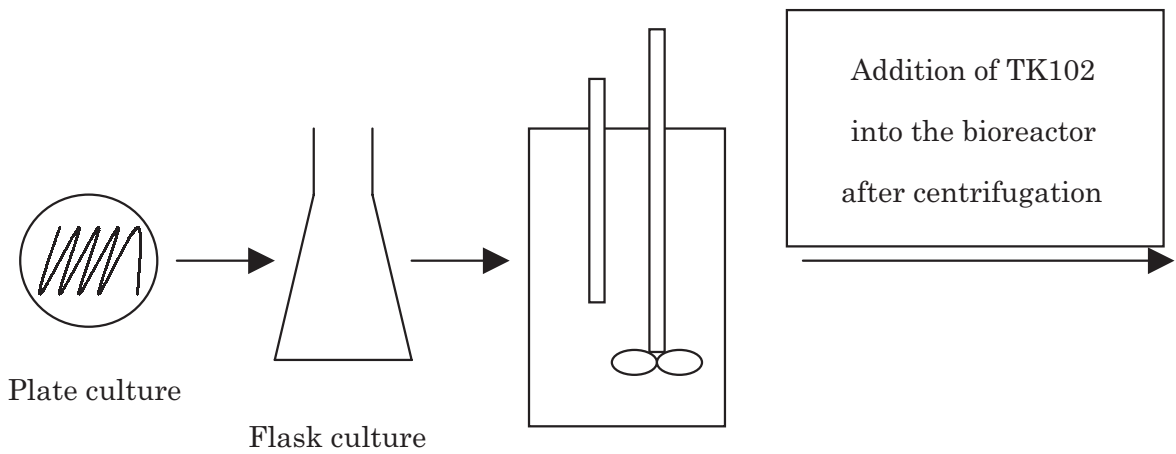

10L Jar fermentor culture

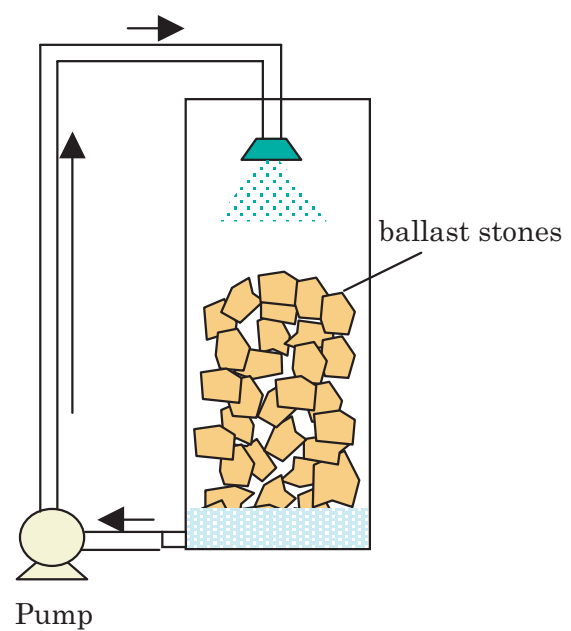

Fig. 3 TK102 biodegradation test flow for PCB treated ballast stones and the test bioreactor 


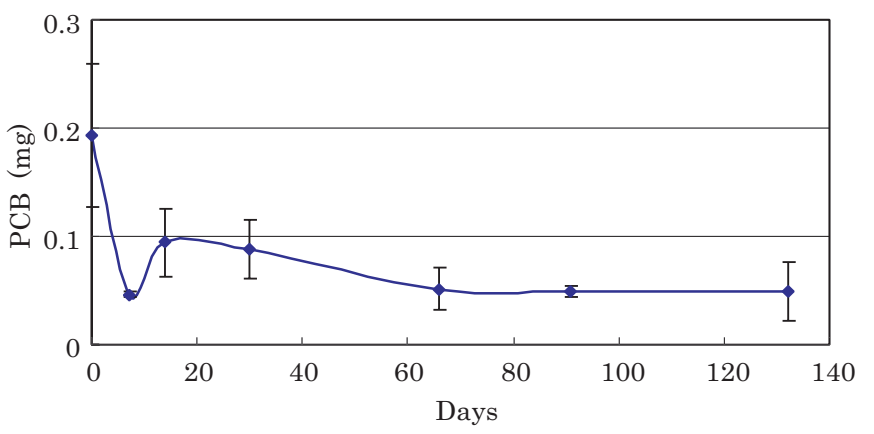

Fig. 4 PCB reduction in ballast stones after TK102 microorganism treatment

\subsubsection{Further Work}

According to these results, the biodegradation by PCB-degrading bacterium, TK102, could be used to decompose PCB in the ballast stones which were contaminated by railway accidents. But it also suggests that there is a limit in the PCB degrading ability of this system. The degradation ability would not last more than two months.

We started a second experiment to confirm these results. We are also planning to evaluate the safety of this bioremediation system by the biological ecotoxicological safety tests that are introduced in this report.

\section{Conclusions}

In this paper we reported several biological safety tests and bioremediation research on the PCB-contaminated ballast stones. Since PCB on the surface of ballast stones was reduced after the treatment with PCB-degrading microorganisms, TK102, this biodegradation system might be an efficient system to degrade PCB in contaminated ballast stones. A combination of biological safety tests and this biodegradation system might lead us to a solution for railway environmental safety.

The "Stockholm Convention on Persistent Organic Pollutants" (UNEP: United Nations Environment Programme) says that we must dispose of PCB completely by 2028 . This means that we must reduce and eliminate toxic chemicals, like PCB, that easily accumulate in the environment and remain for a long time. We are groping with these kinds of problems in the railway environment, from the point of view of global trends. It is inevitable that environmental problems will be addressed globally in the railway fields in the near future.

\section{Acknowledgment}

We are grateful to Mr. Yoshinori Hiraoka, Tokyo Institute of Technology, for allowing us to quote the flow cytometry data.

\section{References}

1) "Guide to producing environmental indicators for the railways," Draft for UIC Workshop, 2001.

2) Ikehata, M., Koana, T., Suzuki, Y., Shimizu, H., Nakagawa, M.: "Mutagenicity and co-mutagenicity of static magnetic fields detected by bacterial mutation assay," Mutation Research, Vol. 427, pp. 147-156, 1999.

3) Nakasono, S., Ikehata, M., Koana, T., Saiki, H.: “A 50 $\mathrm{Hz}, 14 \mathrm{mT}$ magnetic field is not mutagenic or co-mutagenic in bacterial mutation assays," Mutation Research, Vol. 471, pp. 127-134, 2000.

4) Hiraoka, Y., Yamada, T., Kimbara, K.: "Flow cytometric analysis on cell cycle of a PCB-degrading bacterium Comamonas testosteroni TK102," Fifth International Symposium on Environmental Biotechnology, Abstracts, p. 177, 2000.

5) Hund, K., and Traunspurger, W.: "Ecotox-evaluation strategy for soil bioremediation exemplified for a PAHcontaminated site," Chemosphere, Vol. 24, pp. 371390, 1994.

6) Shimura, M., Hayakawa, T., Mukerjee-Dhar, G., Fukuda, M., and Kimbara, K.: "Characterization of polychlorinated biphenyl degradation in a fermentor by Comamonas testosteroni strain TK102," Japanese Journal of Water Treatment Biology Vol. 34, No. 1, pp. 57-65, 1998 . 